\title{
Scintillating fiber based in-vivo dose monitoring system to the rectum in proton therapy of prostate cancer: A Geant4 Monte Carlo simulation
}

\author{
Biniam Tesfamicael ${ }^{1}$, Stephen Avery², Paul Gueye', Donald Lyons1, Mahadevappa Mahesh ${ }^{3}$ \\ ${ }^{I}$ Department of Physics, Hampton University, Hampton, Virginia, USA. \\ ${ }^{2}$ Department of Radiation Oncology, University of Pennsylvania, Philadelphia, Pennsylvania, USA. \\ ${ }^{3}$ Deparment of Radiology and Radiological Sciences, John Hopkins University, Baltimore, Maryland, USA.
}

Received February 04, 2014; Revised March 24, 2014; Accepted March 25, 2014; Published Online March 26,2014

\section{Original Article}

\begin{abstract}
Purpose: To construct a dose monitoring system based on an endorectal balloon coupled to thin scintillating fibers to study the dose to the rectum in proton therapy of prostate cancer. Method: A Geant4 Monte Carlo toolkit was used to simulate the proton therapy of prostate cancer, with an endorectal balloon and a set of scintillating fibers for immobilization and dosimetry measurements, respectively. Results: A linear response of the fibers to the dose delivered was observed to within less than $2 \%$. Results obtained show that fibers close to the prostate recorded higher dose, with the closest fiber recording about one-third of the dose to the target. A $1 / \mathrm{r}^{2}$ ( $\mathrm{r}$ is defined as center-to-center distance between the prostate and the fibers) decrease was observed as one goes toward the frontal and distal regions. A very low dose was recorded by the fibers beneath the balloon which is a clear indication that the overall volume of the rectal wall that is exposed to a higher dose is relatively minimized. Further analysis showed a relatively linear relationship between the dose to the target and the dose to the top fibers (total 17), with a slope of $(-0.07 \pm 0.07)$ at large number of events per degree of rotation of the modulator wheel (i.e., dose). Conclusion: Thin $(1 \mathrm{~mm} \times 1$ $\mathrm{mm})$, long $(1 \mathrm{~m})$ scintillating fibers were found to be ideal for real time in-vivo dose measurement to the rectum during proton therapy of prostate cancer. The linear response of the fibers to the dose delivered makes them good candidates as dosimeters. With thorough calibration and the ability to define a good correlation between the dose to the target and the dose to the fibers, such dosimeters can be used for real time dose verification to the target.
\end{abstract}

Keywords: Prostate Cancer; Proton therapy; Endorectal Balloon; Scintillating Fibers; Geant4 Monte Carlo; Rectal Wall; Hadron Therapy

\section{Introduction}

Prostate cancer is the second leading cause of cancer related death in men, with estimated deaths of about 29,720 for 2013. ${ }^{1-4}$ A number of treatment options are available for prostate cancer patients. The most commonly used radiation therapy modalities include: Brachytherapy implants ${ }^{5-7}$, in-

Corresponding author:

Biniam Yohannes Tesfamicael; Department of Physics, Medical

Physics Program, Hampton University, Virginia, USA.

Email: biniam.yt@gmail.com

Cite this article as:

Tesfamicael BY, Avery S, Gueye P, Lyons D, Mahesh M. Scintillating fiber based in-vivo dose monitoring system to the rectum in proton therapy of prostate cancer: A Geant4 Monte Carlo simulation. Int J Cancer Ther Oncol 2014; 2(2):02024.

DOI: $10.14319 /$ ijcto.0202.4 tensity modulated radiation therapy (IMRT) $)^{8-13}$, and proton therapy. ${ }^{14-17}$ Due to the characteristic Bragg peak and physical properties of the proton beam, it possesses the ability to penetrate deep before stopping in the medium. ${ }^{18}$ Sharp lateral penumbra of the beam also allows sparing of the critical structures adjacent to the tumor volume. ${ }^{19}$ The formation of the Bragg peak in the depth dose distribution and the ability to spread the dose out using a modulator wheel to generate a spread out Bragg peak (SOBP) allows one to highly localize the dose delivered to the target. This gives the advantage of sparing much of the healthy tissue around the target, especially in the distal region. Hence, proton beam therapy is an attractive option for the treatment of cancer. The ability to geometrically conform the dose to the target, however, should be accompanied with stringent requirements in the target localization to avoid error in the dose delivered. The 
two main sources of uncertainties for this are patient setup and organ motion. During prostate cancer therapy, bladder and rectum emptying and/or filling are the main contributors to the organ motion. ${ }^{20,21}$ Research studies showed that such uncertainty can result in the introduction of prostate displacement in the inferior-superior (IS), anterior-posterior (AP), and right-left (RL) directions. Huang et al, ${ }^{13}$ for instance, measured a maximum displacement in the AP (6.8 $\mathrm{mm}$ anteriorly to $4.6 \mathrm{~mm}$ posteriorly) and in the SI (3.5 $\mathrm{mm}$ superiorly to $6.8 \mathrm{~mm}$ inferiorly) directions. Such displacements are large enough to introduce a remarkable error in the dose delivered to the prostate, especially when proton beams are used to treat the tumor.

A number of techniques were devised to minimize the uncertainty introduced by organ motion in the treatment of prostate cancer. The use of an endorectal balloon, currently practiced by a number of cancer centers, was found to greatly reduce the prostate motion by internally immobilizing it. ${ }^{20}, 22$ This technique reduces the prostate displacement to less than $1 \mathrm{~mm}$ according to the study results from McGary et al. $^{22}$ In addition to internally immobilizing the prostate, researchers observed some additional benefits from the use of an endorectal balloon. First, it reduces greatly the overall volume of the rectal wall exposed to a higher dose. ${ }^{20,23} \mathrm{Se}-$ cond, the balloon can be used to give a good prediction of the prostate location. ${ }^{24}$ Most of the currently utilized balloons have an embedded fiducial marker that can be easily identified even in poor quality images. In the presence of a good reproducibility in the placement of the balloon, since it is also used during the simulation computed tomography (CT) scan, fiducial markers (instead of bonny landmarks) can be utilized for patient setup and prostate organ location. ${ }^{24}$ Third, some researchers took advantage of the use of an endorectal balloon to develop an in-vivo dose measurement tool for the rectum. ${ }^{25,26}$ This can be performed by the use of an appropriate dosimeters attached to the balloon to record the dose at the surface of the balloon, which is in contact with the inner rectal wall.

In this work, the Geant4 Monte Carlo toolkit ${ }^{27,}{ }^{28}$ was used to simulate the proton therapy of prostate cancer using an endorectal balloon as an internal immobilization device. Thin scintillating fibers were attached to the balloon for real time in-vivo dose measurement to the surface of the balloon. The characteristic properties that scintillating fibers possess such as water equivalency, temperature and pressure independence, fast response time etc. makes them ideal dosimetric tools for this task. ${ }^{29-33}$ The fact that they can be fabricated in any size and shape also makes them good candidates as they can be prepared in small sizes for a better resolution. This can also make the fibers less intrusive as far as the patient is concerned in clinical applications.

\section{Methods and Materials}

The Hadrontherapy Geant4 example application was used as a reference to design the simulation described in this work. ${ }^{34}$ The modulator wheel that spreads out the Bragg peak to generate an SOBP was modified to accommodate high energy proton beams. A spherical geometry of diameter $2.9 \mathrm{~cm}$, which is made up of a soft tissue equivalent material, G4_TISSUE_SOFT_ICRP, obtained from the National Institute of Standards and Technology (NIST) database ${ }^{35}$, was introduced to simulate the prostate. Geometries for the endorectal balloon as well as the thin long scintillating fibers $(1 \mathrm{~mm} \times 1 \mathrm{~mm} \times 1 \mathrm{~m})$ laying on the top surface and beneath the balloon were also introduced and their placements are detailed in Figure 2.

The beam line was designed with a simplified geometry to mimic the beam line of the Hampton University Proton Therapy Institute (HUPTI). ${ }^{36}$ Hence, a basic difference exists between the beam line in the simulation design developed and the one at HUPTI.

\section{Results}

The Geant4 based simulation developed was validated against dedicated data acquired at HUPTI for a range of 257 $\mathrm{mm}$ and a modulation of $13 \mathrm{~mm}$. The SOBP generated for a $200 \mathrm{MeV}$ proton beam is compared to the measured data from HUPTI in Figure 1 (arbitrary units were used to normalize the data in the SOBP region).

The simulation data are in good agreement with the measured data. From Figure 1, the analysis of the data shows that at the beginning of the SOBP and at the dose fall off regions, the data points match within $4 \%$ and $3 \%$, respectively. At the end of the SOBP, however, there is a remarkable difference between the two data sets. This is due to the combined effect of the beam line (differences in the geometry) and the modulator wheel residual. At the distal 10\% dose, a depth difference of $2.5 \mathrm{~mm}$ was observed. The noticeable difference between the measured and the simulation data at the entrance dose is due to the effect of the different amount of secondary particles generated, a consequence of the beam line discrepancy between the simplistic simulation and the actual beam line at HUPTI.

In the simulation, a total of 34 fibers were placed around the endorectal balloon for dosimetry (17 atop and 17 beneath). The placement of the fibers around the balloon is shown in Figure 2. 


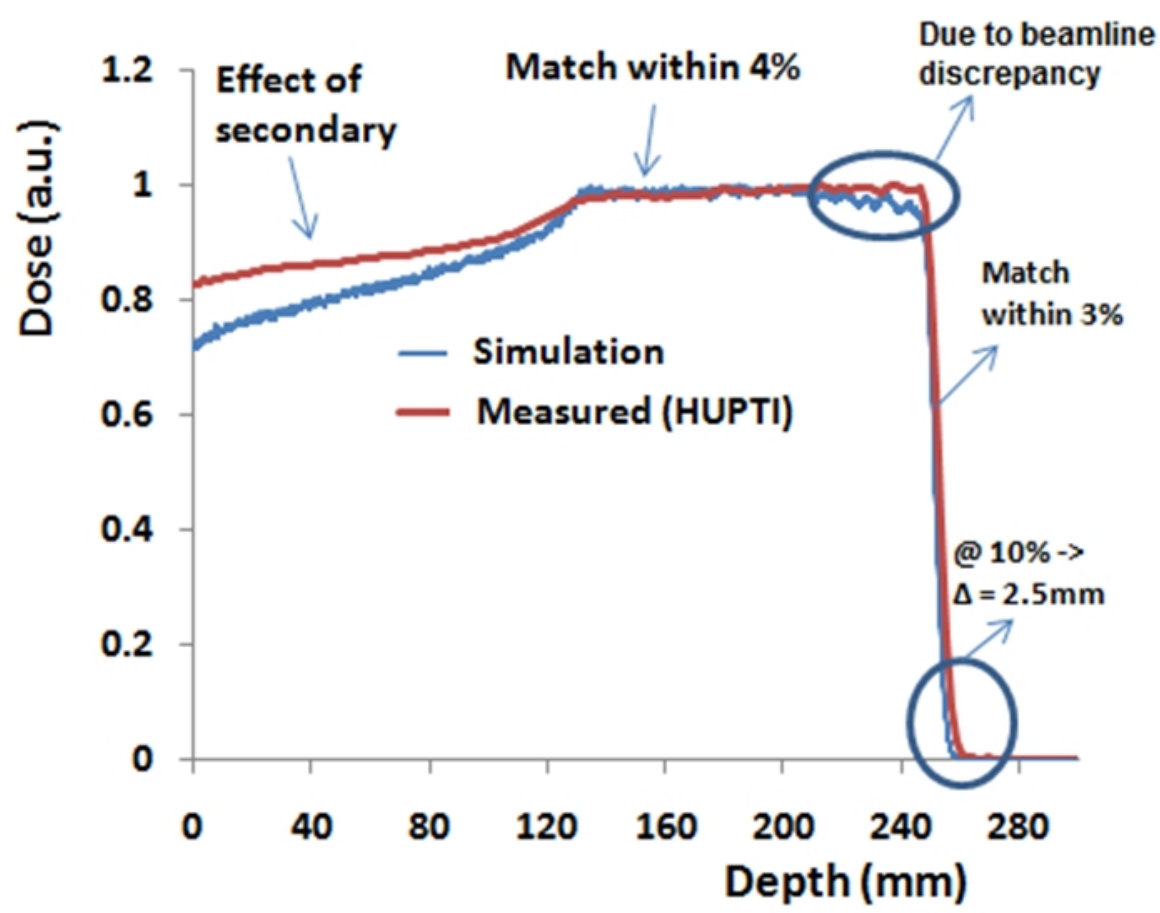

FIG. 1: Comparison of the SOBP distribution from the Geant4 simulation and experimental data for a range of 257 mm and a modulation of 13 $\mathrm{mm}$. The distributions are normalized in the SOBP region.

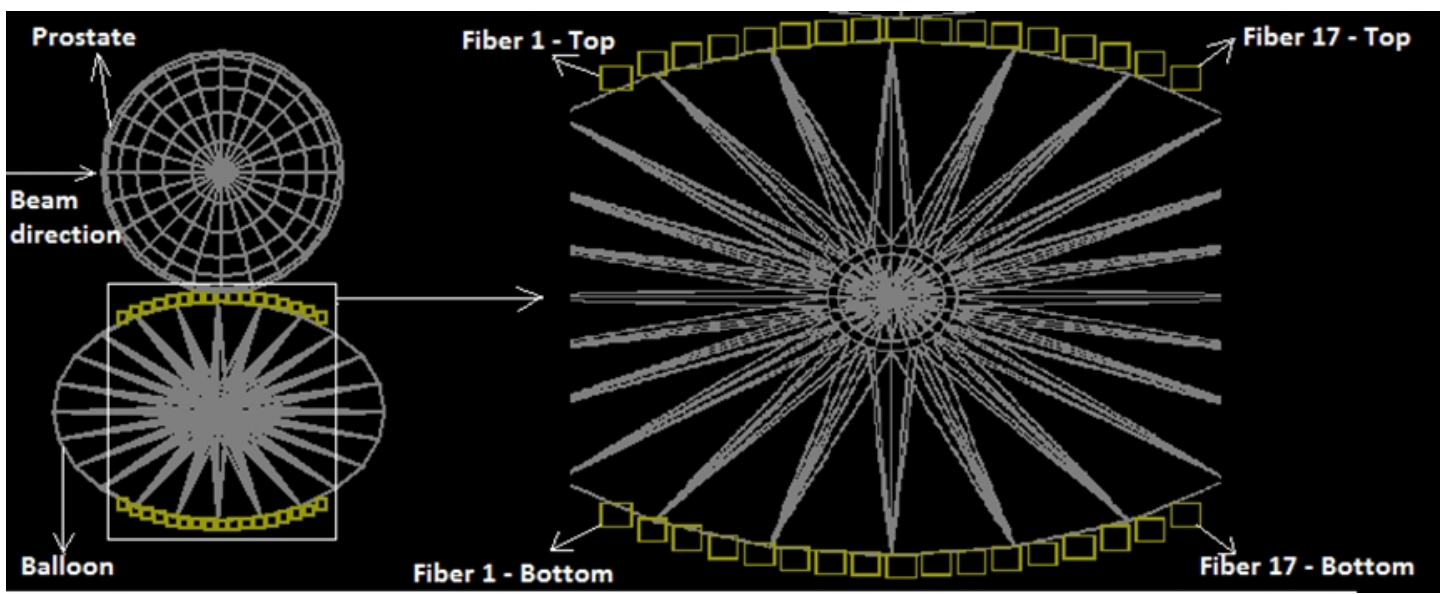

FIG. 2: The placement of the scintillating fibers around the balloon. The prostate is located on the top of the balloon, with the top fibers sandwiched in-between.

As shown in Figure 2, the fibers were laid on the surface of the balloon, with the fiber ID running from 1 to 17 from left to right. The beam direction is from left to right, irradiating the whole prostate. At the end of the simulation run, the energy deposited in all 34 fibers were recorded by requiring a cut in the volume of interest. Therefore, the knowledge of the geometrical location of each fiber is an important step.
To acquire such information, the beam propagation axis ( $\mathrm{x}$-axis) was aligned with the center of the balloon. The plot of the interaction points for the fibers in the Y-X (transverse-longitudinal, cross sectional view) and Y-Z (transverse, beam eye view) planes is shown in Figure 3. 

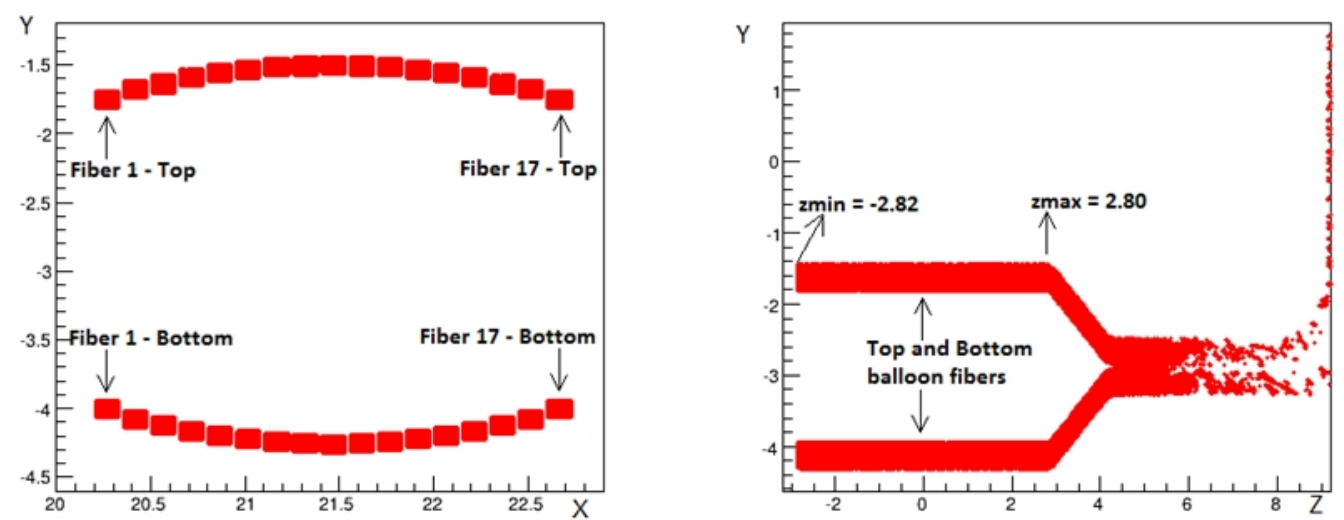

FIG. 3: The cross-sectional and beam eye view of the plot of the interaction points of the radiation in the top and bottom fibers.

The left panel of Figure 3 shows the cross-sectional view of the 34 fibers around the balloon (17 top and 17 bottom), with the beam momentum in the positive $\mathrm{X}$ direction. The $\mathrm{X}$ $\left(\mathrm{X}_{\min }, \mathrm{X}_{\max }\right)$ and $\mathrm{Y}\left(\mathrm{Y}_{\min }, \mathrm{Y}_{\max }\right)$ boundaries for each fiber were extracted from the scatter distributions. The length of the fibers along the surface of the balloon was acquired from the readings of $Z_{\min }$ and $Z_{\max }$. These six coordinate values gave the volume of the individual fibers that are effectively collecting the information on the surface of the balloon. These values were subsequently used to calculate the energy deposited above and below the balloon.

In irradiating the prostate, $200 \mathrm{MeV}$ protons were used with the modulator wheel rotating from $0^{0}$ to $359^{\circ}$ in steps of $1^{0}$, for a total $360^{\circ}$ to cover the entire prostate. The geometry for the prostate was placed at a depth of $19.5 \mathrm{~cm}$ in a water filled tank of $36 \mathrm{~cm} \mathrm{x} 22 \mathrm{~cm} \mathrm{x} 24 \mathrm{~cm}$ from the beam entrance surface. The simulation was run for four different number of events per degree of modulator wheel rotation: 500, 1000, 2000 and 3000 corresponding to a dose in $\mu \mathrm{Gy}$ to the prostate of $0.11,0.21,0.43$, and 0.65 , respectively. The total integrat- ed energy deposited in each fiber inside the geometrical volume of interest specified in Figure $\mathbf{3}$ was recorded and is plotted in Figure 4.

The energy deposited in the bottom fibers, which corresponds to the inner posterior wall of the rectum, was negligibly small compared to the energy deposited in the top fibers as well as inside the prostate: the maximum value recorded was $3.99 \mathrm{MeV}$ for the central and for the 3000 events per degree of modulator rotation run. The energy deposited in the prostate as well as the total energy deposited in the top 17 fibers are given in Table 1 . The table also shows the number of protons delivered in each setup. One can observe that the number of protons used is very small compared to the number required in the clinic to deliver a dose of 1.8 Cobalt Gray Equivalent (CGE) to a $\mathrm{Kg}$ of water, which is of the order of $10^{10}$. This is the dose per fraction most of the time prescribed for prostate cancer patients at HUPTI. In the simulation, the dose delivered was of the order of a few $\mu \mathrm{Gy}$ due to computational limitations.

\begin{tabular}{cccc}
\hline \hline $\begin{array}{c}\text { Number of events } \\
\text { Per degree }\end{array}$ & $\begin{array}{c}\text { Total number of events } \\
\text { (protons) }\end{array}$ & $\begin{array}{c}\text { Total energy deposited in } \\
\text { the prostate (MeV) }\end{array}$ & $\begin{array}{c}\text { Total energy deposited in the top } \\
\text { fibers }(\mathrm{MeV})\end{array}$ \\
\hline 500 & $1.80 \times 10^{5}$ & $8,696.22$ & 111.51 \\
1000 & $3.60 \times 10^{5}$ & $16,667.64$ & 263.73 \\
2000 & $7.20 \times 10^{5}$ & $34,381.29$ & 504.38 \\
3000 & $1.08 \times 10^{6}$ & $51,881.16$ & 812.04 \\
\hline \hline
\end{tabular}

TABLE 1: Energy deposited in the prostate and the top 17 fibers during the irradiation of the prostate for the four different settings used in the Geant4 simulation.

The calculation of the energy deposited per unit volume $(\mathrm{MeV} / \mathrm{cc})$ gave 4062 and 1214 for the prostate and the top middle fiber (Fiber ID 9), respectively. These correspond to an absorbed dose of $0.65 \mu \mathrm{Gy}$ and $0.19 \mu \mathrm{Gy}$ for the prostate and the fiber, respectively. This result showed that the section of the rectal wall represented by Fiber ID 9 is pushed by the inflated balloon to the high dose region, receiving about a third of the dose deposited in the prostate. As one moves away from the middle fiber towards the frontal and distal regions, the value of the energy deposited per unit volume decreases dramatically. For Fiber ID 1 (frontal region) and Fiber ID 17 (distal region) the values were 517 (factor of 1/8) and 428 (factor of 1/9), respectively, corresponding to an absorbed dose of approximately $0.08 \mu \mathrm{Gy}$ and $0.07 \mu \mathrm{Gy}$ to the frontal and distal fibers, respectively. In the actual treatment of a prostate cancer patient, the anterior section of 
the rectal wall is usually included in the PTV. Consequently, the top Fiber ID 9 will be inside the PTV, receiving the prescribed dose. Hence, this fiber can be used for real time dose verification to the PTV during irradiation. In the simulation, however, all the top fibers were kept outside of the field, a reason for the absorbed dose to be lower for the fibers than that of the prostate. Similar results were obtained for the other simulation runs. The corresponding plot of the energy deposited versus the Fiber IDs is shown in Figure 4. The energy deposited as a function of the number of events per degree of modulator rotation (i.e., dose), for the prostate and the total energy deposited to the 17 top fibers, is shown in Figure 5.

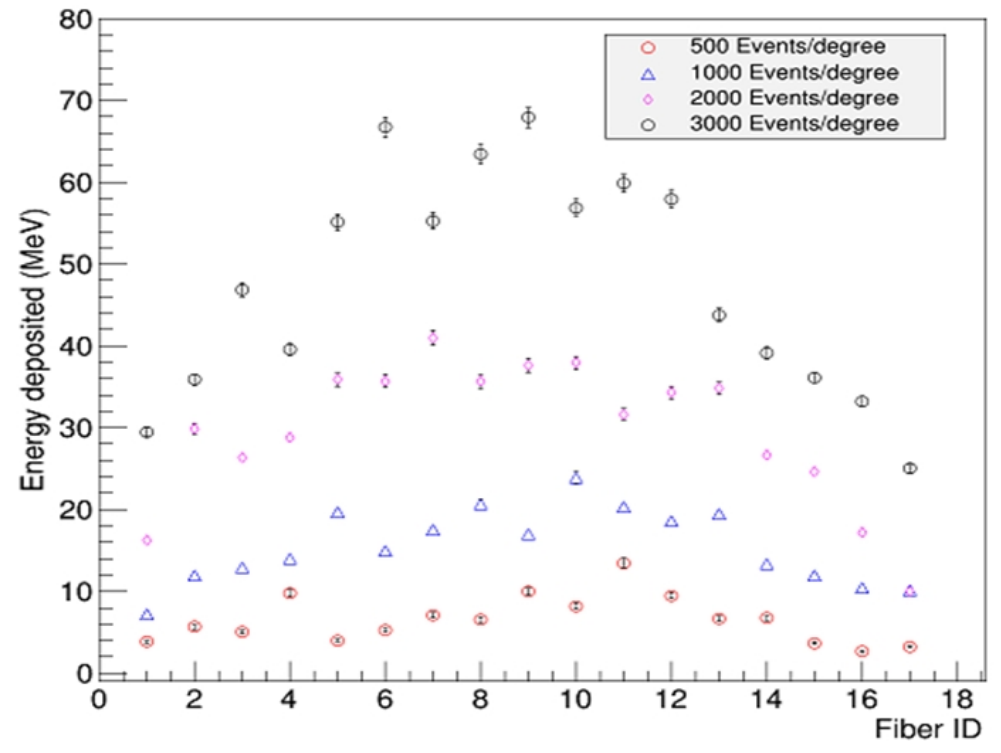

FIG. 4: A plot of the energy deposited in the top fibers as a function of the fiber ID for the different number of events per degree of modulator wheel rotation.

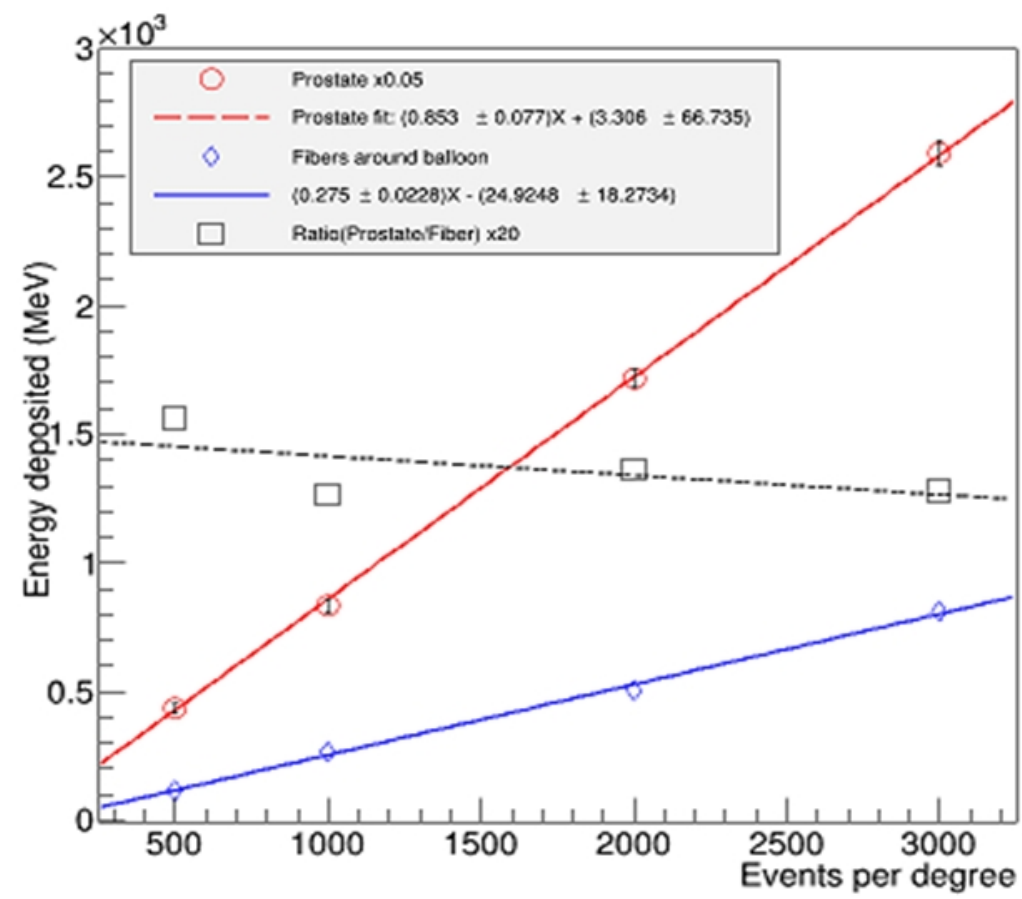

FIG. 5: A plot of the total energy deposited in the top 17 fibers as well as in the prostate as a function of the events per degree of rotation (i.e., dose). The ratio of the energy deposited in the prostate to the total energy in the top 17 fibers is also shown. The energy in the prostate is scaled down by 0.05 , while the ratio is scaled up by 20 for clarity. 


\section{Discussion}

From the results obtained, one observes that increasing the number of events per degree of rotation (i.e., dose) increases the energy deposited inside the fibers. In Figure 4, it can be observed that the energy deposited is largest around the middle fibers, especially for the central (Fiber ID 9), which is the closest to the prostate. In moving to the frontal (Fiber ID 1) and distal (Fiber ID 17) regions, the energy deposited decreases as $1 / \mathrm{r}^{2}$ ( $\mathrm{r}$ is defined as center-to-center distance between prostate and fiber), which implies that the overall rectal wall volume exposed to higher radiation is smaller. This result is in good agreement to the results obtained by other researchers. ${ }^{20,}{ }^{23}$ In the actual planning of the treatment, the top section of the rectum is usually enclosed within the planning target volume (PTV). Hence, the Fiber ID 9, located at the top middle, is usually enclosed within the PTV receiving the prescribed dose. With appropriate calibration, this fiber can be used to monitor the dose delivered to the PTV.

Further analysis of the data showed that there is also a linear response of the scintillating fibers to the radiation dose delivered to the prostate, with a precision of less than $2 \%$ as show in Figure 5: slope of $0.853 \pm 0.077 \mathrm{MeV} /$ events per degree for the prostate and $0.275 \pm 0.023 \mathrm{MeV} /$ events per degree for the fibers. This result is one of the characteristic properties studied by a number of other researchers that make the fibers ideal as a dosimetric tool. ${ }^{29-33}$ Moreover, the ratio of the energy deposited in the prostate to the total energy deposited in the top 17 fibers gets relatively constant, especially for larger number of events (slope of $-0.07 \pm 0.07$ $\mathrm{MeV}$ per $\mathrm{Gy}$ to the prostate). Once a clear relationship is defined between the dose to the prostate and the dose to the fibers, those fibers can be used as dose verification tools to the target.

\section{Conclusion}

In this work, the use of an endorectal balloon coupled to thin scintillating fibers was studied as a potential dose monitoring system in proton therapy of prostate cancer. Based on the results obtained, the energy deposited in the fibers placed atop the balloon was observed to decrease as $1 / \mathrm{r}^{2}$ as one goes to the distal and proximal ends from the central fiber. In addition, the total energy deposited inside the 17 fibers located beneath the balloon near the posterior section of the rectal wall was $0.27 \%$ of the energy deposited in the top middle fiber (Fiber ID 9) alone. These results indicate that the top section of the rectal wall is pushed to the high dose region while the overall volume of the rectal wall exposed to a higher dose is very small. Further analysis of the data shows that the ratio of the dose deposited in the prostate to the total dose deposited in the top 17 fibers is relatively flat with a slope of $-0.07 \pm 0.07$, especially at large number of events. Hence, with appropriate calibration, these fibers can be used to give a precise prediction of the dose delivered to the prostate, opening the capability of an in-vivo dose monitoring system during irradiation. An experimental setup will be designed based on the simulation presented herein. The results will be presented in a forthcoming paper.

\section{Acknowledgment}

This work was partly supported by the Department of Energy - National Security Administration under award number DE-NA0000979.

\section{Conflict of interest}

The authors declare that they have no conflicts of interest. The authors alone are responsible for the content and writing of the paper.

\section{References}

1. American Cancer Society. Cancer Facts and Figures 2013. American Cancer Society, Atlanta, GA.

2. Crawford ED. Epidemiology of prostate cancer. Urology 2003; 62:3-12.

3. Gronberg H. Prostate cancer epidemiology. Lancet 2003; 361:859-64.

4. Cartwright LE, Suchowerska N, Yin Y, et al. Dose mapping of the rectal wall during brachytherapy with an array of scintillating dosimeters. Med Phys 2010; 37:2247-55.

5. Mazeron R, Bajard A, Montbarbon X, et al. Permanent 125I-seed prostate brachytherapy: early prostate specific antigen value as a predictor of PSA bounce occurrence. Radiat Oncol 2012; 7:46.

6. Nag S, Beyer D, Friedland J, et al. American Brachytherapy Society (ABS) recommendations for transperineal permanent brachytherapy of prostate cancer. Int J Radiation Oncology Biol Phys 1999; 44:789-99.

7. Porter AT, Blasko JC, Grimm PD, et al. Brachytherapy for prostate cancer. CA Cancer J Clin 1995; 45:165-78.

8. Hong TS, Ritter MA, Tome WA, Harari PM. Intensity-modulated radiation therapy: emerging cancer treatment technology. $B r \quad J$ Cancer 2005; 92:1819-24.

9. Zelefsky MJ, Fuks Z, Leibel SA. Intensity-modulated radiation therapy for prostate cancer. Semin Radiat Oncol 2002; 12:229-37.

10. Ezzell GA, Galvin JM, Low D, et al. Guidance document on delivery, treatment planning, and clinical implementation of IMRT: report of the 
IMRT Subcommittee of the AAPM Radiation Therapy Committee. Med Phys 2003; 30:2089-115.

11. Zelefsky MJ, Fuks Z, Happersett L, et al. Clinical experience with intensity modulated radiation therapy (IMRT) in prostate cancer. Radiother Oncol 2000; 55:241-9.

12. Cahlon $\mathrm{O}$, Hunt $\mathrm{M}$, Zelefsky MJ. Intensity-modulated radiation therapy: supportive data for prostate cancer. Semin Radiat Oncol 2008; 18:48-57.

13. Huang E, Dong L, Chandra A, et al. Intrafraction prostate motion during IMRT for prostate cancer. Int J Radiat Oncol Biol Phys 2002; 53:261-8.

14. Slater JD, Rossi CJ Jr, Yonemoto LT, et al. Proton therapy for prostate cancer: the initial Loma Linda university experience. Int J Radiat Oncol Biol Phys 2004; 59:348-52.

15. Slater JD, Rossi CJ Jr, Yonemoto LT, et al. Conformal proton therapy for early-stage prostate cancer. Urology 1999; 53:978-84.

16. Moyers MF, Pouliot J, Orton CG. Point/Counterpoint. Proton therapy is the radiation treatment modality for prostate cancer. Med Phys 2007; 34:375-8.

17. Slater JD, Yonemoto LT, Rossi CJ Jr, et al. Conformal proton therapy for prostate canrcinoma. Int $J$ Rad Oncl Biol Phys 1998; 42:299-304.

18. Ulmer W. Notes of the editorial board on the role of medical physics in radiotherapy. Int J Cancer Ther Oncol 2013;1:01014.

19. Rana S, Singh H. Impact of heterogeneities on lateral penumbra in uniform scanning proton therapy. Int J Cancer Ther Oncol 2013; 1:01026.

20. Wachter S, Gerstner N, Dorner D, et al. The influence of a rectal balloon tube as internal immobilization device on variations of volumes and dose volume histograms during treatment course of conformal radiotherapy for prostate cancer. Int $J$ Radiat Oncol Biol Phys 2002; 52:91-100.

21. Adamson $\mathrm{J}, \mathrm{Wu}$ Q. Prostate intrafraction motion assessed by simultaneous kilovoltage fluoroscopy at megavoltage delivery I: clinical observations and pattern analysis. Int J Radiat Oncol Biol Phys 2010; 78:1563-70.

22. McGary JE, Teh BS, Butler EB, Grant W 3rd. Prostate immobilization using a rectal balloon. J Appl Clin Med Phys 2002; 3:6-11.

23. Cho JH, Lee CG, Kang DR, et al. Positional reproducibility and effects of a rectal balloon in prostate cancer radiotherapy. J Korean Med Sci 2009; 24:894-903.

24. Ciernik IF, Baumert BG, Egli P, et al. On-line correction of beam portals in the treatment of prostate cancer using an endorectal balloon device. Radiother Oncol 2002; 65:39-45.
25. Archambault L, Briere TM, Pönisch F, et al. Toward a real-time in vivo dosimetry system using plastic scintillation detectors. Int $J$ Radiat Oncol Biol Phys 2010; 78:280-7.

26. Hardcastle N, Cutajar DL, Metcalfe PE, et al. In vivo real-time rectal wall dosimetry for prostate radiotherapy. Phys Med Biol 2010; 55:3859-71.

27. Agostinelli S, et al., Geant4 - A simulation toolkit. Nuclear Instruments and Methods in Physics Research 2003; A506:250-303.

28. Allison J, et al., Geant4 developments and applications, IEEE Transactions on Nuclear Science 2006; 53:270-278.

29. Knoll GF. Radiation Detection and Measurement. John Wiley \& Sons Inc New York, 2000.

30. Beddar AS, Mackie TR, Attix FH. Water-equivalent plastic scintillation detectors for high-energy beam dosimetry: I. physical characteristics and theoretical considerations. Phys Med Biol 1992; 37:1883-900.

31. Smith DL, Polk RG, and Miller TG. Measurement of the response of several organic scintillators to electrons, protons and deuterons. Nucl Instrum Meth 1968; 64:157-66.

32. Beddar AS, Mackie TR, Attix FH. Water-equivalent plastic scintillation detectors for high-energy beam dosimetry: II. Properties and measurements. Phys Med Biol 1992; 37:1901-13.

33. Gooding TJ and Pugh HG. The response of plastic scintillators to high-energy particles. Nucl Instrum Meth 1960; 7:189-92.

34. Cirrone GAP, Cuttone G, Guatelli S, Lo Nigro S, Mascialino B. Implementation of a new monte carlo simulation tool for the development of a proton therapy beam line and verification of the related dose distributions. IEEE Trans. Nucl Sci 2005; 52:262-5.

35. NIST physical measurements laboratory. Available from http://physics.nist.gov/PhysRefData/Star/Text/AST AR.html. [Accessed date: 10/29/2012]

36. Available from http://www.hamptonproton.org/ [Accessed date: 02/03/2014] 